\title{
SIG-Blocks: Tangible Game Technology for Automated Cognitive Assessment
}

\begin{abstract}
This paper presents the SIG-Blocks system developed for automated cognitive assessment via tangible geometric games (TAG-Games). Computerized game administration and real-time cognitive and behavior assessments were realized by wireless self-synchronization in communication, decentralized hybrid-sensing, assembly and motion detection, and graphical visualization. The measurable performance data included time and accuracy at each manipulation step, overall speed of manipulative motions, and the total number of rotational motions. For preliminary evaluation, three types of TAG-Games were designed: TAG-Game ${ }^{\mathrm{A}}$ for assembly, TAG-Game ${ }^{\mathrm{S}}$ for shape matching, and TAG-Game ${ }^{\mathrm{M}}$ for memory. As a part of the game design, a computational measure of play complexity was defined for each TAG-Game based on the geometric properties and the number of blocks in the item. An evaluation with 86 participants assessed both reliability of the TAG-Game items using split-half and test-retest reliability tests and validity of the proposed complexity measures by comparing the results with three subtests of the Wechsler Adults Intelligence Scale 4th Edition (WAIS-IV), i.e. Block Design (BD), Matrix Reasoning (MR), and Digit Span (DS). The high reliability coefficients show that TAG-Games are reliable. Regarding validity, correlations were found between TAG-Game ${ }^{\mathrm{A}}$ and $\mathrm{BD}$ and between TAG-Game ${ }^{\mathrm{S}}$ and MR. Behavioral analysis also showed that the TAG-Game performance was positively correlated with the manipulation speed, but not correlated with the total number of rotations applied to the blocks.
\end{abstract}

Keywords: Sensor-embedded blocks, Tangible user interface, Wireless sensor network, Cognitive assessment 


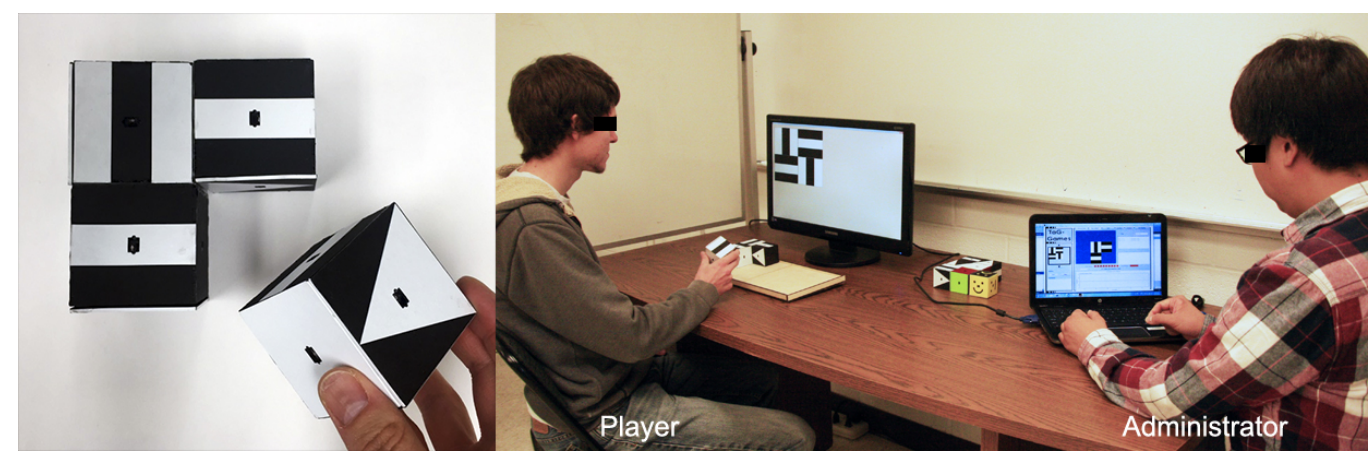

Figure 1: Overview of SIG-Blocks and TAG-Games. A player is given a set of SIG-Blocks and plays computerized TAG-Games while the game items are displayed on a computer screen. The administrator adminiters the tet and monitors the player's performance in real time. The administrator may administer the games from a remote location.

\section{Introduction}

Skilled cognitive performance is a highly complex process that involves 3 perception, memory, reasoning, problem solving, and executive functions 4 (Sparrow \& Davis, 2000). Cognitive skills work in concert to produce gen5 eral intellectual and learning capabilities (Kulp, 1999; Short \& Weissberg6 Benchell, 1989; Schweikardt, 2006). The ability to measure these compo7 nents efficiently is essential for better understanding of the cognitive impairs ments associated with many disorders, including brain injuries, intellectual 9 disabilities, dementia, psychiatric/mental disabilities, and other neurological and age-related conditions (Tatemichi, Desmond, Stern, Paik, Sano, \& Bagiella, 1994; Christensen, Griffiths, MacKinnon, \& Jacomb, 1997; Pe2 tersen, Smith, Waring, Ivnik, Kokmen, \& Tangelos, 1997). Cognitive as3 sessment has evolved into a widely diversified subject. The Wechsler tests 14 are among the earliest and most widely accepted instruments of cognitive assessments (Silverman, Miezejeski, Ryan, Zigman, Krinsky-McHale, \& Urv, 2010; Strauss, Sherman, \& Spreen, 2006). The Raven's Progressive Matrices (RPM) is another widely used test, which is often accepted when verbal constraints are in place or the clinician chooses to test non-verbal intelligence (Raven \& Court, 1998). RPM relies on a series of perceptual and analytical reasoning problems presented in a matrix format (Bors \& Stokes, 1998).

Sometimes, cognitive assessments use physical objects in order to track motor responses as well as cognitive processing (Lezak, 2004). Geometric blocks are employed in many such assessments because they are a benchmark 
for observing manipulation patterns and developmental transformations. As such, they are often employed as an evaluation tool for understanding how children develop spatial cognition and fine motor skills (Pehoski, Henderson, \& Tickle-Degnen, 1997). For example, the Block Design subtest in the Wechsler tests employs a set of cubes to examine how quickly and accurately the examinee assembles the cubes to match the item configuration. Achievement of certain developmental milestones can be captured by performance on manipulative tasks, such as piling and assembling blocks and inserting objects in holes (Lockman, 2000; Polatajko \& Cantin, 2005). Object manipulation tasks using geometric blocks can also be used for cognitive rehabilitation of patients with cognitive and/or fine-motor skill deficiencies caused by traumatic brain injury (TBI), stroke, cerebral palsy, Huntington's disease, and dementia (Millis, Rosenthal, Novack, Sherer, Nick, Kreutzer, High, \& Ricker, 2001; Fork, Bartels, Ebert, Grubich, Synowitz, \& Wallesch, 2005; Lund \& Nielsen, 2011; Wright, Rosenbaum, Goldsmith, Law, \& Fehlings, 2008; Willingham, Koroshetz, \& Peterson, 1996). Using geometric blocks in cognitive assessment, or using any other physical objects, requires the administrator to closely observe the examinee's performance while administering the test and recording measurable data (e.g. accuracy and speed for each test item). Compared to a paper-pencil test, this process can be more labor-intensive and error-prone.

This paper presents SIG-Blocks (Sensor-Integrated Geometric Blocks), developed for tangible, computerized assessments of cognitive skills using physical blocks (Fig. 1). The computer-based games using SIG-Blocks are called TAG-Games (Tangible Geometric Games). Each SIG-Block detects the overall manipulative motions applied by the player and the assemblies made with neighboring blocks. The system, involving a set of SIG-Blocks and a host computer, features independent wireless communication between each SIG-Block and the computer using radio frequency, and block-to-block communication using infrared (IR) signals. For reliable and efficient blockto-computer (global) and block-to-block (local) communications, distributed wireless sensor network (DWSN) algorithms, including self-synchronization and hybrid-sensing techniques, were implemented. Further, computational measures of play complexity were incorporated in TAG-Games to quantify the difficulty associated with game items. This feature, once fully validated, can be used to personalize the test items to address individual/group differences. SIG-Blocks and TAG-Games were designed for automated, realtime, and remote assessment of cognitive skills, as well as hand-eye coordi- 
nation skills. These were aimed at addressing the following limitations that are common in traditional assessment methods: 1) high cost due to clinician/administrator time, 2) limited amounts and types of measurable data by a human administrator, 3) subjectivity and human measurement errors, 4) limited accessibility to professional services for people living in hard-toreach areas, and 5) difficulty in addressing individual differences in cognitive skills, age, and developmental status, because of lack of flexibility in test items.

As a first step in evaluating the clinical utility of this technology and establishing the reliability and validity of the outcome measures, we designed three types of TAG-Games, each aimed at measuring specific domains of cognitive skills via tangible object manipulation: TAG-Game ${ }^{\mathrm{A}}$ for assembling multiple blocks to match the given assembly configuration, TAG-Game ${ }^{\mathrm{S}}$ for identifying a missing block in assembly configurations, and TAG-Game ${ }^{\mathrm{M}}$ for memorizing a sequence of block images. For each TAG-Game, we defined a computational measure of play complexity, $C$, to quantify difficulty associated with each game, based on geometric complexity and the number of blocks. This measure, once fully evaluated, can be used for personalizing the test items tailored for each individual. Our evaluation study focused on testing the following two hypotheses: H1. Performances in TAG-Games are correlated to the scores from standardized cognitive assessment tests, and H2. Computational measures of play complexity defined for TAG-Games properly reflect the difficulty of the test items. To evaluate our hypotheses, we used the Wechsler Adult Intelligent Scale - 4th Edition (WAIS-IV), which is the most widely used instrument for cognitive assessment. In particular, we selected three subtests in WAIS-IV: Block Design (BD), Matrix Reasoning (MR), and Digit Span (DS). Each of these subtests is considered to be closely related to a TAG-Game by test design and target measures.

\section{Related Work}

Several technology-embedded block systems have been developed for a broad range of education, entertainment, and research purposes. Table 1 lists these systems and compares them with SIG-Blocks, in terms of communication capabilities, embedded sensors, measurable data, and data display methods. One such system, AlgoBlock was developed for children to learn programming via tangible manipulation of the blocks (Suzuki \& Kato, 1995). The AlgoBlocks facilitate interactive learning of computer programming by 
allowing users to write their own problems. This is accomplished by giving each AlgoBlock its own special semantics. Tangible Programming Bricks with a built-in microprocessor were developed for children to learn programming language via tangible manipulation of bricks (McNerney, 1999). Electronic Blocks, a similar system that allows the user to build simple computer programs by stacking blocks with embedded electronic circuits, was also designed for young children (Wyeth \& Wyeth, 2011). Cognitive Cubes is another system developed for cognitive and constructional assessment (Sharlin, Itoh, Watson, Kitamura, Sutphen, \& Liu, 2002). These cubic blocks are equipped with male-female connectors for forming a 3-D assembly and network topology with a base cube that is wired to the host computer. The roBlock system, which teaches advanced engineering concepts such as kinematics, feedback, and distributed control, also employs blocks to teach the user (Schweikardt \& Gross, 2006). The working principle of roBlocks is similar to that of Electronic Blocks. Cubelets is a modular robotic kit consisting of sensor, logic, and actuator blocks (www.modrobotics.com). By assembling different types of Cubelets, a user can build a robot with the desired communication and sensing capabilities. Learning Cube is a digitally augmented block system, enriched with LED displays and an internal speaker (Terrenghi, Kranz, Holleis, \& Schmidt, 2006). It is capable of gesture recognition by using an accelerometer in creating an interactive learning interface. Sifteo is another sensor-embedded block system with a small liquid-crystal display (LCD) screen on its top face (Merrill, Sun, \& Kalanithi, 2012). The Sifteo blocks can sense and communicate with each other and detect tilt and shake motions. Lastly, the Multi-Agent System supports educational games for children with autism (Alers \& Barakova, 2009). It displays its active state through color and light intensity by detecting the status of neighboring blocks with infrared (IR) communication.

In considering the effectiveness of each block system for automated, realtime assessment of cognitive skills, three criteria are considered: sensibility, scalability, and communicability. The blocks must be equipped with sensors that can detect the physical motions applied to each of them and the assembly configurations among multiple blocks. The number of blocks used in the test/game must be scalable to support 2-dimensional (2D) and 3-dimensional (3D) assemblies with varying difficulty. Bi-directional communication between the blocks and a host computer and among the blocks will support real-time sensor data acquisition, as well as assembly detection among the assembled blocks. The SIG-Blocks system has some unique features that 
satisfy all of these criteria. Only Cognitive Cubes and Sifteo support both block-to-computer and block-to-block communications, but neither of them supports block-to-computer wireless communication. The SIG-Blocks system was operated in a fully decentralized manner without any hierarchical or master-slave structure, such as that used in Cognitive Cubes, and is therefore fault-tolerant. In addition, most existing systems were designed for tangible gaming or educational purposes, but they lack the necessary recording functions, which are essential for assessment function. SIG-Blocks with TAG-Games were specifically designed to provide feedback on the player's performance and behavior via real-time, wireless, and bidirectional communications. Only Learning Cube demonstrates block-to-computer wireless communication capability based on radio frequency (RF) (Terrenghi et al., 2006).

Among the existing systems, Sifteo has the most features comparable to those of SIG-Blocks in terms of sensing and communication capabilities. Sifteo adopts a gateway called Sifteo-base, a special block which is connected to a host computer using a USB cable for downloading new games. In addition, this base is responsible for wireless communication with the rest of the Sifteo blocks. The SIG-Blocks system differs from Sifteo in the following ways: 1) the low-cost infrared (IR) communication method for block-to-block communication, 2) direct wireless communication between each block and a host computer without requiring a gateway, 3) continuous sensor-data collection from each block, and 4) applicability for both 2D and 3D construction tasks. First, while Sifteo adopts four IrDA transceivers, SIG-Block uses six pairs of IR LEDs and a photo transistor. Second, bi-directional wireless communication exists between each SIG-Block and a host computer. The computer can wirelessly control the mode of the blocks for different games and each block sends the collected sensor data, including orientation and assembly information, to the computer. Third, continuous data can be collected for assessment. Lastly, the cube design of SIG-Blocks enables 3D construction games. However, Sifteo may still be more suitable for games under some circumstances. Although the LCD display on Sifteo limits its workspace to 2D, it renders Sifteo more suitable for entertainment and educational applications than the plane images used in SIG-Blocks. As the current version of the SIG-blocks was designed for cognitive assessment, clear geometric patterns were selected. 


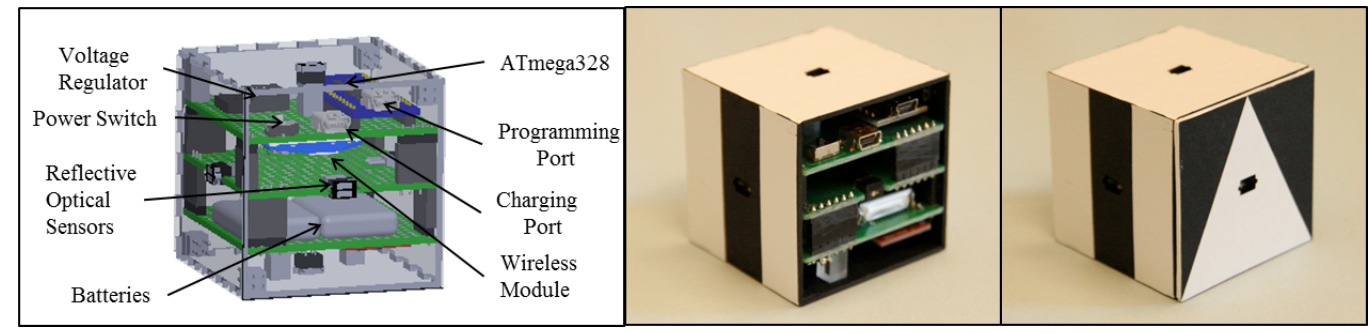

Figure 2: SIG-Blocks with improved hardware design. The programming port and charging port can be easily accessed by detaching the covers. The outer cover is made with mat-board (non-toxic, light, and durable against impact).

\section{SIG-Blocks}

SIG-Blocks are interactive creation blocks designed to be used as a tangible and computerized interface for various games (i.e. TAG-Games) that involve tangible manipulations of the blocks. This section describes the design considerations, technical specifications, and wireless communication strategies embedded in the blocks.

\subsection{Design Considerations \\ Sensibility}

One of the main considerations is the measurable data. When geometric blocks are used in an educational or clinical test, the administrator records the time and accuracy of the tasks completed by the player. Therefore, the quantity and quality of the measurable data are limited by the human capability. To automate the measurement process, while potentially improving the quantity and quality of the data, the blocks must be capable of sensing the player's action applied to them as well as synthesizing the actions. To do so, the block must be capable of sensing the orientation and the accelerations applied while being manipulated by hands, and of detecting neighboring blocks when assembled. Various sensors can realize these functions. For example, accelerometers, gyroscopic sensors, liquid tilt sensors, and/or magnetometers can provide the block's orientation state. In addition, simple optical sensors or mechanical contact sensors may be sufficient for face-to-face interaction among the blocks.

\section{Scalability}

SIG-Blocks are intended to be used for creating universal 2-D and 3-D shapes, and therefore should be scalable to form a large structurally stable 
configuration when assembled. Among the various 3-D shapes, such as cubes, cylinders, spheres, cones, and tetrahedrons, cubic structures enable efficient and stable stacking and provide an adequate number of degrees of freedom without any additional docking support. With respect to perception, recognizing the rotations of a cube is intuitive, because the axes of the coordinate system that represents the cube are orthogonal to the faces, and therefore, the orientation of the cube is easily understood when the top and bottom faces are parallel to the tabletop. With this universal design, a number of tasks would be available by reprogramming the functions to interact with new task environments. Identical cubes would be more desirable because of their common geometric properties and shared communication protocols and electronics. Homogeneity in block design also allows for replacement of any broken block by a new one.

\section{Communicability}

The blocks should perform data measurement on board, wirelessly communicate with other blocks to detect assembly, and transfer data with the remote data storage, i.e. the host computer. When multiple blocks are used in a wireless sensor network (WSN), the communication subsystem is the main infrastructure on which the WSN is constructed, and hence the communication protocols affect the overall sensing accuracy and reliability. To ensure a large and reliable communication subsystem, several technical issues need to be addressed. Such issues may include synchronization, data traffic, and non-deterministic lag time between the transmitter and the receiver. Without utilizing the master and slave mechanism, synchronization becomes challenging; therefore, the self-synchronization method, which continuously transmits and receives data and shifts bits by a given rule at each phase, must be implemented. This leads to a rapid massive data collection which generates significant data traffic when the host computer collects the data through a single receiver. Therefore, the data should be optimized in terms of size without loss of meaningful information and sent efficiently by an event-driven method. Also, the non-deterministic lag time during wireless data transmission should avoid data collision in fast data transfer.

\subsection{Technical Description}

\subsubsection{Design and specifications}

SIG-Blocks feature 1) block-to-block communication using infrared (IR) optical sensors, 2) bidirectional block-to-computer communication using an 
XBee module, and 3) light and compact packaging (Fig. 2). Compared to the previous prototype presented in (Jeong, Kerci, \& Lee, 2010), the block's size was reduced from 2.7 inches to 2.05 inches in length along each side, and the weight from $355 \mathrm{~g}$ to $82 \mathrm{~g}$. Each block contains a triaxial accelerometer (ADXL335), a microprocessor (ATmega328), six reflective optical sensors, and an XBee wireless module. It is powered by two 3.7 Volt, $350 \mathrm{mAh}$ polymer lithium-ion batteries, which are rechargeable through a USB connector. It takes less than 30 minutes to recharge each block and the operating time is approximately 3.5 hours.

Core electronic components were embedded in three stacks of printed circuit board (PCB) layers and were encased in a cubic block made of matboard. The outer surfaces were covered by six distinctive geometric images, shown in Fig. 3. These images were selected to represent 1-, 2-, and 4-fold rotational symmetry, where each pair had the same shape, but with reversed colors (Jeong et al., 2010). Retaining the core module intact, cover layers can be customized for different purposes. The reflective optical sensor installed at the center of each block's surface detects the reflected IR signal. By combining the data from the optical sensors and the accelerometer, assembly configurations of the blocks can be reconstructed for real-time feedback. Also, local communication between the assembled blocks was enabled through these optical sensors with protocols proposed in Section 3.3. These optical sensors may require periodic replacement. Female sockets were used for positioning these optical sensors to facilitate replacement.

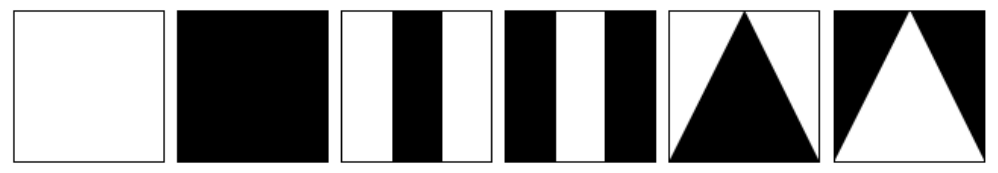

Figure 3: Six distinctive images with 1-, 2-, and 4- fold rotational symmetry.

\subsubsection{Measurable data and sensing capability}

Each block can detect its relative position, orientation, and elapsed time after each assembly made by the player. When used in geometric games, such as matching an assembly configuration or identifying a missing piece in a puzzle, the blocks can also determine whether they are placed correctly or incorrectly. Six embedded optical sensors, a triaxial accelerometer, and a 
timer in the microprocessor were used to determine the accuracy of manipulation, the time for each manipulation step, and possibly behavioral characteristics such as impulsive or repetitive motion. Each block sends ten packets of data to a host computer wirelessly. The first packet is the ID of the block and the next six packets are binary optical sensor data corresponding to the assembly status of each block's surface. The last three packets correspond to acceleration data along $x, y$, and $z$ directions and their values range between $-1.0 \mathrm{~g}$ to $1.0 \mathrm{~g}$.

\subsection{Wireless Communication Strategies}

The SIG-Blocks system features the following communication capabilities: IR-based inter-block (local) communication and bidirectional block-tocomputer (global) communication using an XBee module, based on the distributed wireless sensor network (DWSN) scheme. To avoid resource conflict, deadlocking, and to establish reliable wireless communication, two strategies were adopted: 1) synchronization techniques (self-synchronization) to initiate local communication, and 2) efficient network topology (decentralized hybrid-sensing network) with even-driven data input for optimal global communication.

\subsubsection{Self-synchronization in local communication}

SIG-Blocks utilize self-synchronization for initializing local communication between two adjacent blocks. Two communication ports, $\mathbf{A}$ and $\mathbf{B}$, are considered fully synchronized if $\mathbf{B}$ listens while $\mathbf{A}$ talks, and $\mathbf{A}$ listens while $\mathbf{B}$ talks. However, as shown in Fig. 4, signals transmitted by $\mathbf{A}$ and B may mutually conflict when they both talk or listen at the same time. The self-synchronization method uses an internal clock, transmitted pulse, and received pulse to determine the port that needs to listen while the other talks. This bit-shift strategy was performed as follows, for port A (Jeong \& Lee, 2016):

$$
\begin{gathered}
I_{A}=[1,2,3, \cdots, 30,31,32] \\
T_{A}=[0,0, \cdots, 0,0,1,1, \cdots, 1,1] \\
R_{A}=[*, *, \cdots, *, *, *, *, \cdots, *, *]
\end{gathered}
$$

$I_{A}$ is the 32-bits cyclic bit number index generated by the internal clock, $T_{A}$ is the transmitted binary data, and $R_{A}$ is the received signal from the other port. The pulse train from $T_{A}$ is generated from the IR photo diode according to the bit number of the $I_{A}$. When the two ports, $\mathbf{A}$ and $\mathbf{B}$, are 


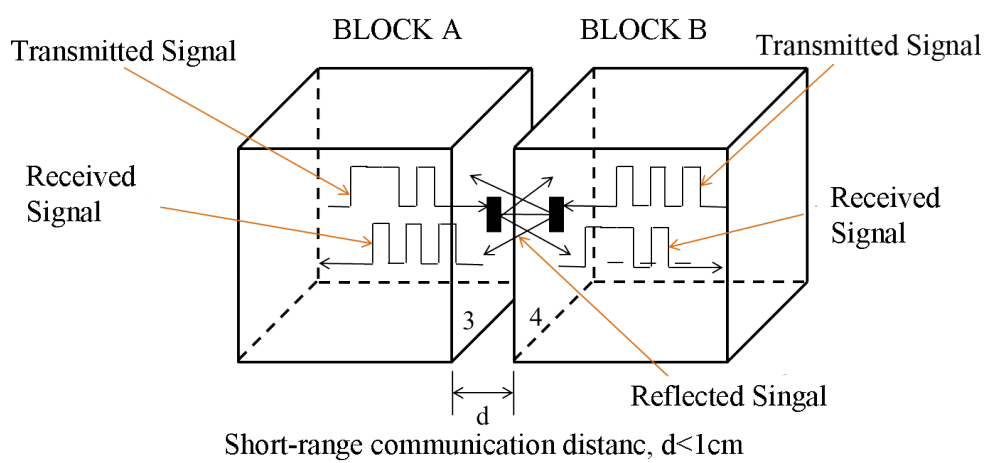

(a)

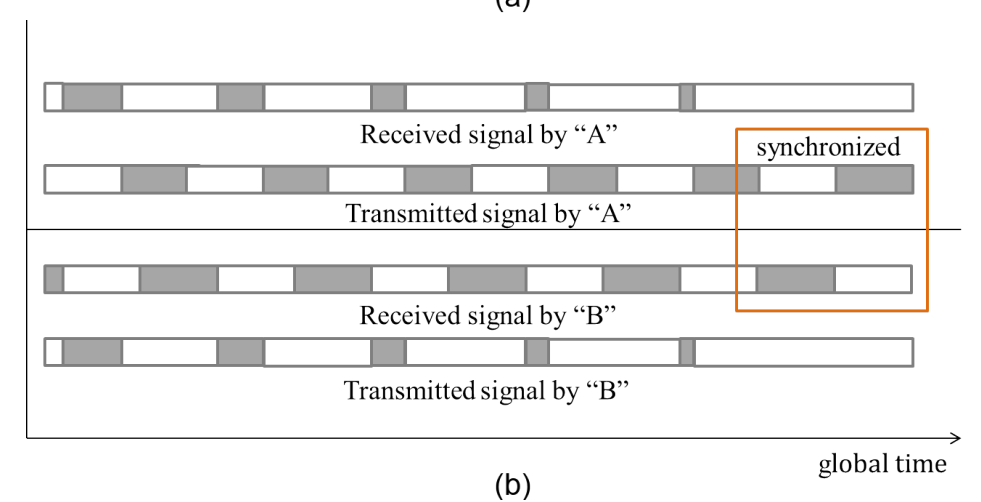

Figure 4: (a) Short-range communication mechanism used in the new SIG-Blocks via IR sensors and (b) self-synchronization scheme between the ports $\mathbf{A}$ and $\mathbf{B}$. The gray bar indicates talking bytes while the white bar refers to listening bytes (Jeong \& Lee, 2016).

within the local communication range, $R_{A}$ receives data from $T_{B}$, as well as reflected signal from $T_{A}$. Due to the pull-up resistors attached to the photo transistors, $R_{A}$ is set as 1 initially or when no IR signals are received, and it changes to 0 when IR input is detected. Decision for shifting, either forward or backward, for synchronization can be made stochastically; however, this process can take a long time. For every 31st bit of the 32 bits (4 bytes), the binary train moves one step forward or two steps forward depending on the received data. If $\sum_{n=b i t 1}^{b i t 8} R_{A}(\mathrm{n})$ is smaller than $\sum_{n=b i t 9}^{b i t 16} R_{A}(\mathrm{n})$, then the pulse train proceeds one step forward, but when it is bigger, the pulse train moves two steps forward. When the two transmitted signals are the same, a random shift is applied to each port, so that it makes partial synchronization. When the synchronization is completed, IR communication data packets are replaced in the last two bytes of $T_{A}$. Fig. 4 illustrates this process. 


\subsubsection{Decentralized hybrid-sensing network (DHSN)}

To avoid data traffic when multiple blocks are used in the game, the system utilizes a DHSN. While low-power local communication is always on, the data transfer between each block and the host computer is triggered only when an event occurs. During play, most of the blocks will remain stationary excepting the few that are being manipulated at the time. The event-driven data transfer is triggered by the acceleration reaching a threshold value, which is determined by the noise density level of the accelerometer. Event-driven data transfer is more effective than periodic data transfer at preventing loss of any significant information. Also, static channel access control was utilized because dynamic control requires both linking and stabilizing time, which has the potential to cause significant time delay in wireless communication.

\section{Algorithm Implementation}

In this section, the proposed wireless communication algorithms are implemented for block assembly detection, motion detection, and visualization for real-time administration and monitoring of the user's performance and behavior.

\subsection{Assembly Detection}
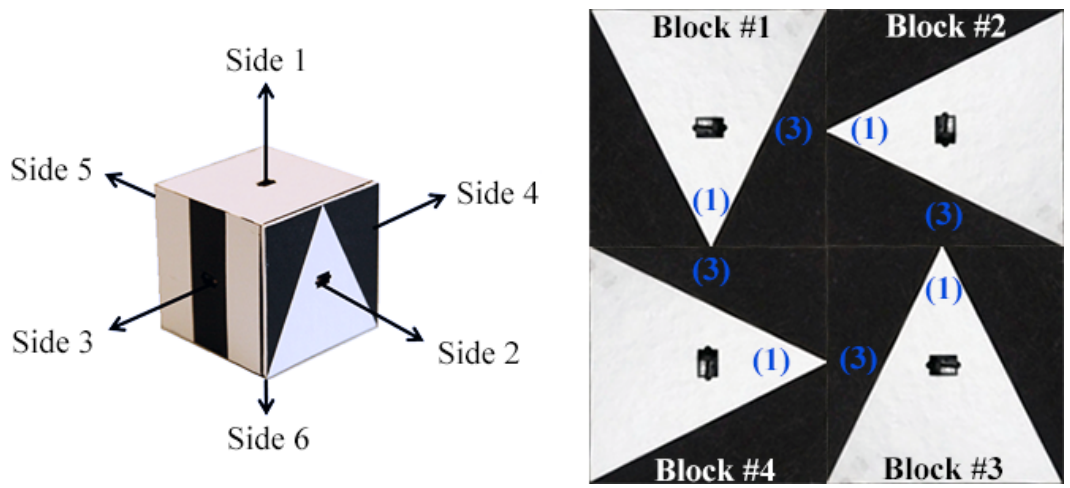

Figure 5: A SIG-Block with six sides, each with a unique ID number, and an example of $2 \times 2$ assembly configuration seen from the top.

When two blocks are assembled, the assembly configuration is captured by a pulse train via local inter-block communication, using IR sensors. The 
pulse train involves the following information: (ID1, SIDE1, TOP1, ID2, SIDE2, TOP2). ID1 and ID2 are the ID numbers of the two blocks assembled, SIDE1 and SIDE2 are the surfaces on the blocks facing each other, and TOP1 and TOP2 are the surfaces of each block that face upward. Considering the low response and signal reading time in the photo transistor and the microcontroller's input port, 1 millisecond per bit for the pulse train was selected. For detecting each assembly, the configuration matrix, $A$, was generated by labeling the block's IDs and side IDs. For the example shown in Fig. 5, $A$ is a $6 \times 6$ matrix consisting of four $3 \times 3$ matrix blocks where each matrix block contains the SIG-Block ID in the $(2,2)$ entry and the four sides information in the $(1,2),(2,1),(2,3)$, and $(3,2)$ entries. Before and after assembly, the changes in $A$ are as follows:

$$
A:=\left[\begin{array}{cccccc}
\star & 0 & \star & \star & 0 & \star \\
0 & 1 & 0 & 0 & 2 & 0 \\
\star & 0 & \star & \star & 0 & \star \\
\star & 0 & \star & \star & 0 & \star \\
0 & 4 & 0 & 0 & 3 & 0 \\
\star & 0 & \star & \star & 0 & \star
\end{array}\right] \rightarrow\left[\begin{array}{cccccc}
\star & 0 & \star & \star & 0 & \star \\
0 & 1 & 3 & 1 & 2 & 0 \\
\star & 1 & \star & \star & 3 & \star \\
\star & 3 & \star & \star & 1 & \star \\
0 & 4 & 1 & 3 & 3 & 0 \\
\star & 0 & \star & \star & 0 & \star
\end{array}\right]
$$

For an $n \times m$ assembly configuration in 2-D, $A \in \mathcal{R}^{3 n \times 3 m}$ consists of $n \times m$ matrix blocks. For 3-D assemblies, $A$ can be defined as a $3-\mathrm{D}$ matrix, or keep the same 2-D matrix format and store the assembly statuses of the top and bottom surfaces on empty spaces, indicated by $\star$ in the above matrix.

\subsection{Motion Detection (Jeong et al., 2010)}

Once the linear acceleration data is transferred from the SIG-Blocks, the data is processed in the host computer to be translated into rotational motions applied to the blocks. To enable the use of a triaxial accelerometer in detecting angular displacements of each block, the noise caused by measurements of hand-operational movements will have to be filtered. The low-cost capacitive accelerometer used in this study featured signal conditioning, a 1-pole low-pass filter, temperature compensation, and g-select which allows for selecting one of the four sensitivities: $1.5 \mathrm{~g}, 2 \mathrm{~g}, 4 \mathrm{~g}$, and $6 \mathrm{~g}$. The highest sensitivity of this accelerometer, $800 \mathrm{mV} / \mathrm{g}$, occurred when $1.5 \mathrm{~g}$ was selected. Three output ports on the accelerometer provided analog acceleration signals along $x-, y$-, and $z$-axes. These analog signals were then transformed to digital signals through the analog-to-digital converter in the microprocessor 

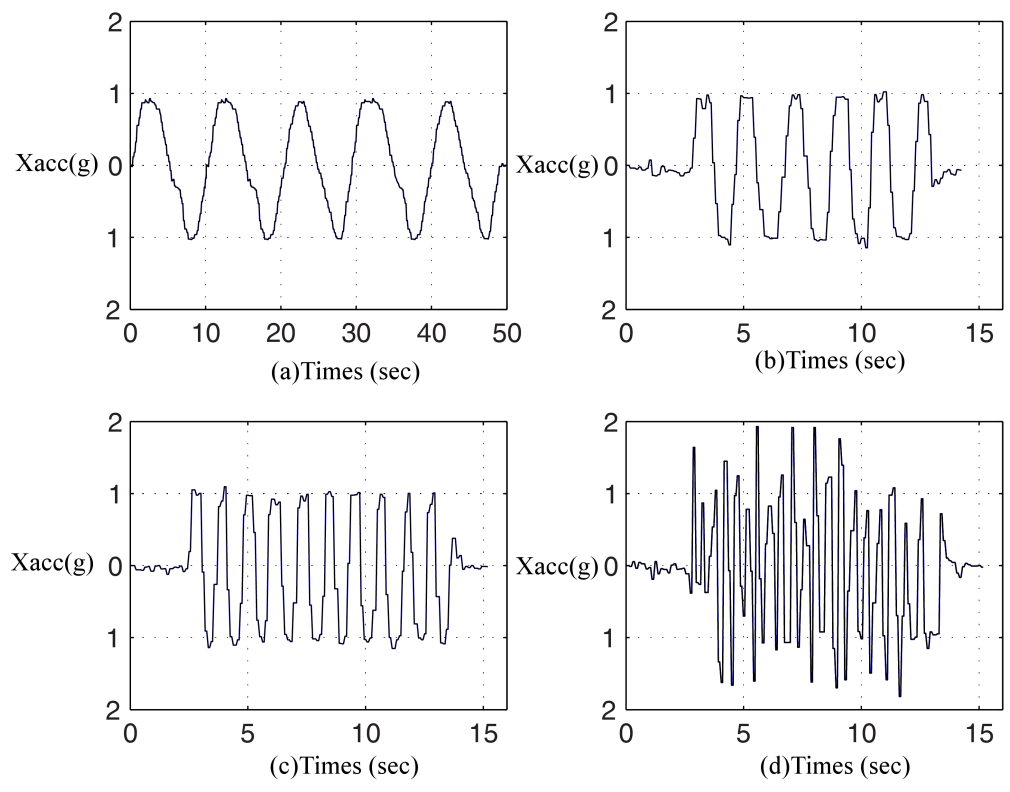

Figure 6: Acceleration along the $x$-axis, $x_{a c c}$, captured from the triaxial accelerometer versus time while rotating about the $y$-axis (Jeong et al., 2010).

(ATmega328). When the accelerometer is in static state, it can detect angular displacements by measuring static accelerations due to gravity, which range between $-g$ and $+g$. Using a triaxial accelerometer, tilting angles along the $x$-axis and $y$-axis can be calculated based on the acceleration data $\left(x_{a c c}, y_{a c c}, z_{a c c}\right)$ by (Wong \& Wong, 2008):

$$
\theta_{\text {pitch }}=\tan ^{-1}\left(\frac{x_{a c c}}{\sqrt{\left(y_{a c c}\right)^{2}+\left(z_{a c c}\right)^{2}}}\right) ; \quad \theta_{\text {roll }}=\tan ^{-1}\left(\frac{y_{a c c}}{\sqrt{\left(x_{a c c}\right)^{2}+\left(z_{a c c}\right)^{2}}}\right) \text {. }
$$

Detecting tilting angles of a moving accelerometer is challenging. To analyze the effect of dynamic acceleration, we conducted a simple block test by manually rotating a SIG-Block $90^{\circ}$ and $-90^{\circ}$ about the $x$-axis repeatedly at different frequencies. Fig. 6a was generated by rotating the block 5 cycles per 50 seconds $(0.1 \mathrm{~Hz})$. Figures $6 \mathrm{~b}-\mathrm{d}$ show the results when the block was rotated at $0.5 \mathrm{~Hz}$ (5 cycles per 10 seconds), $1.0 \mathrm{~Hz}$ (10 cycles per 10 seconds), and $2.0 \mathrm{~Hz}$ (20 cycles per 10 seconds), respectively. The results show 

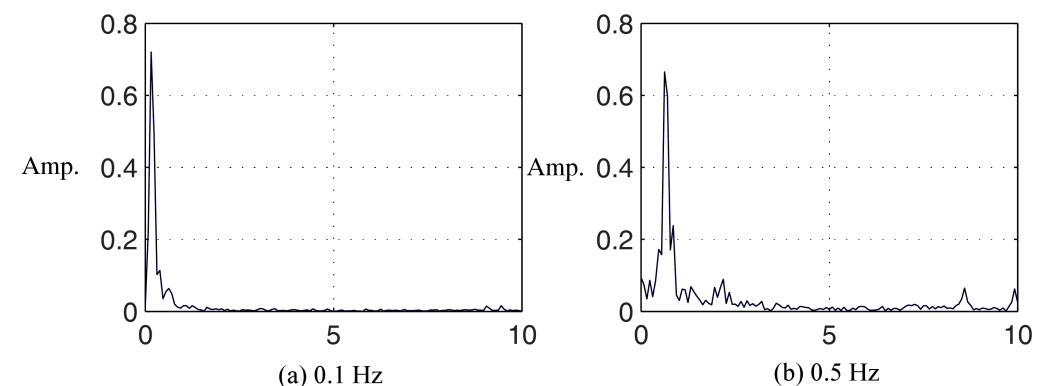

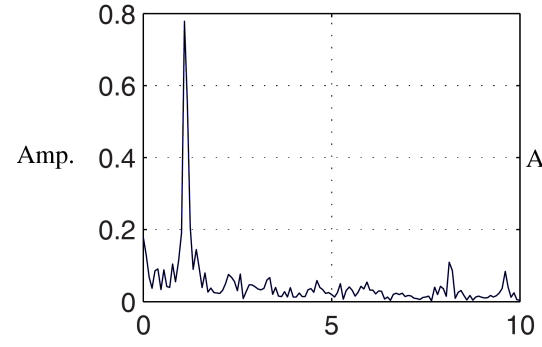

(c) $1.0 \mathrm{~Hz}$

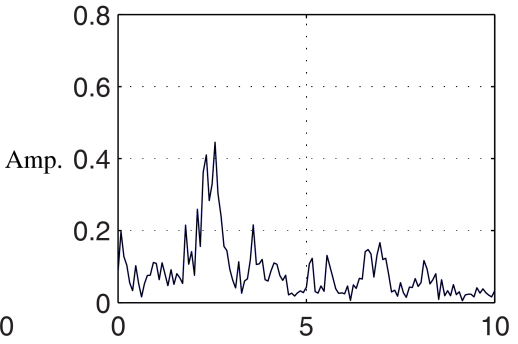

(d) $2.0 \mathrm{~Hz}$

Figure 7: Frequency spectrum of the rotating motions in four different frequencies (Jeong et al., 2010).

that the overall influence of dynamic acceleration is trivial for slow motions $(\leq 1.0 \mathrm{~Hz}$ ), while the error increases at higher frequency domains. This is corroborated by Bernmark's finding that shows that the accelerometer is not influenced by dynamic motion less than $0.75 \mathrm{~Hz}$ (Bernmark \& Wiktorin, 2002). For fast motions, shown in Fig. 6d, the $x_{a c c}$ exceeds $\pm 1 g$ corresponding to $\pm 90^{\circ}$ in the block's orientation because of dynamic acceleration influencing the data. Although every user behaves uniquely and may have different frequency responses, humans have a limited speed and range of motions for manipulating rigid objects as shown in Fig. 7. Therefore, since the SIG-Blocks are expected to be used within low frequency ranges, they can successfully retrieve their rotational information and provide reliable data on how they are manipulated by a player.

\subsection{Graphical User Interface (GUI)}

The GUI was developed based on Microsoft Visual C\# with an open graphics library (OpenGL) for graphical visualization. Tangible games for 


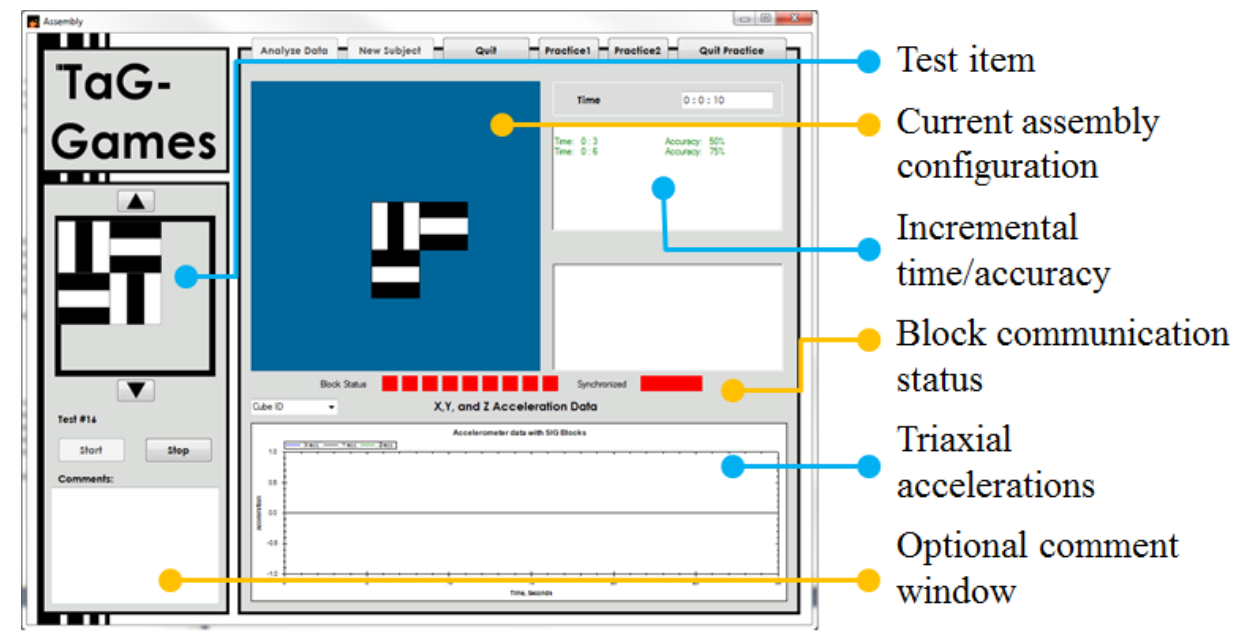

Figure 8: The administrator's GUI that displays test item, measurable data, and block status in real time. The player's GUI simply displays the test item.

cognitive assessment using SIG-Blocks require two GUIs. One of these GUIs serves as a game display for the player, and the other allows the administrator to administer the game and monitor the player's performance in real-time. This monitoring may be conducted remotely, if necessary. The administrator's GUI displays the test/game item, current configuration of the blocks, time and accuracy at each manipulation step, communication status of the blocks used in the game, and triaxial accelerations in real time (Fig. 8). The player's GUI simply displays the test item and/or the current block configuration depending on the game type.

\section{TAG-Games}

Three types of TAG-Games were developed for preliminary evaluation of the SIG-Blocks technology for automated cognitive assessment. The three games are TAG-Game ${ }^{\mathrm{A}}$ for block assembly, TAG-Game ${ }^{\mathrm{S}}$ for shape matching, and TAG-Game ${ }^{\mathrm{M}}$ for sequence memory. Table 2 lists the cognitive skills that are hypothesized to be associated with each game. In addition, each TAGGame item is assigned a value, $C$, that is a computational representation of its play complexity. 


\subsection{TAG-Game ${ }^{A}:$ Assembly}

TAG-Game ${ }^{\mathrm{A}}$ is an assembly construction game in which the user recreates a displayed image using the SIG-Blocks. The displayed image is an arrangement of the geometric shapes on SIG-Block surfaces. The user must rotate and rearrange the blocks to create the displayed pattern. The difficulty and novelty of this task are reflected in the manipulation of the blocks to find the correct face images, the size of the pattern, and the discriminability of the block surface images within the pattern image. The discriminability of the patterns can be decreased, making the task more difficult, by removing the separating lines between the pattern components. This change results in increasing the picture size and making it more prominent in the patterns than the picture of individual block face images. Fig. 9 shows 20 assembly items.

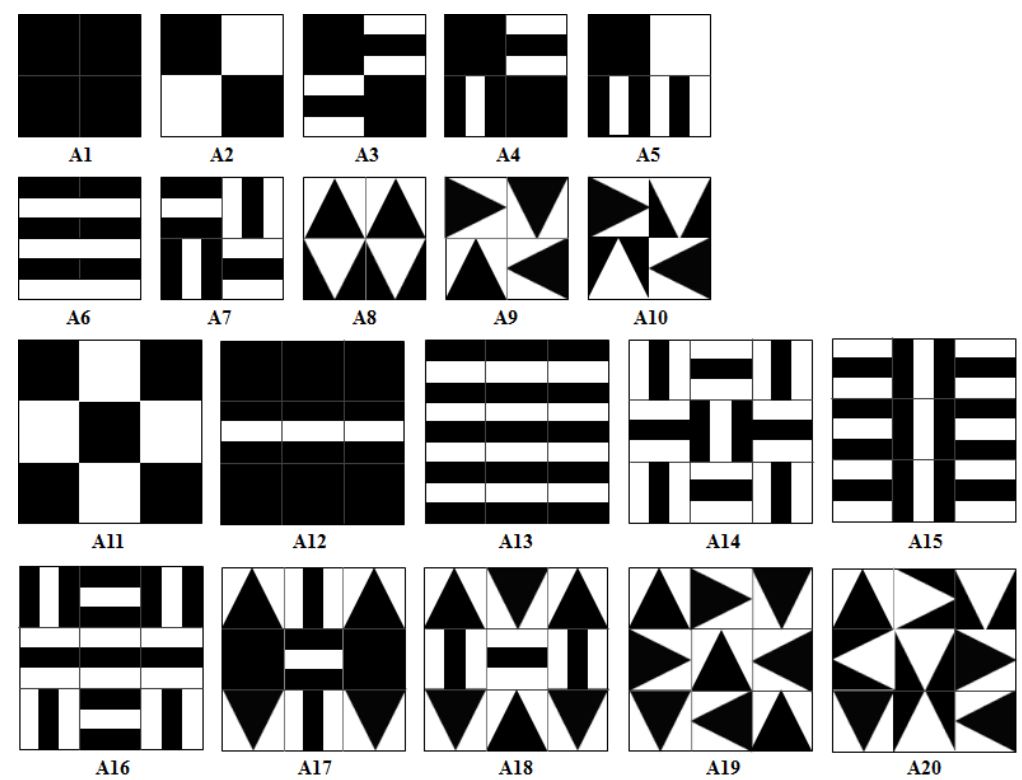

Figure 9: 20 TAG-Game $^{\mathrm{A}}$ items. The lines separating 4 or 9 individual shapes can be removed to potentially increase difficulty. Images as presented in this figure were used in our human-subject evaluation.

\subsection{TAG-Game $e^{S}$ : Shape-Matching}

As shown in Fig. 10, TAG-Game ${ }^{\mathrm{S}}$ consists of 10 items for which an assembly pattern with a missing image is displayed and the participant is 
and accuracy with which the participant can rotate the block to find the correct face image. Working memory is reflected in how well the participant remembers the sequence of images. Finally, attention span is reflected in the participant's ability to maintain focus when the sequences become longer.

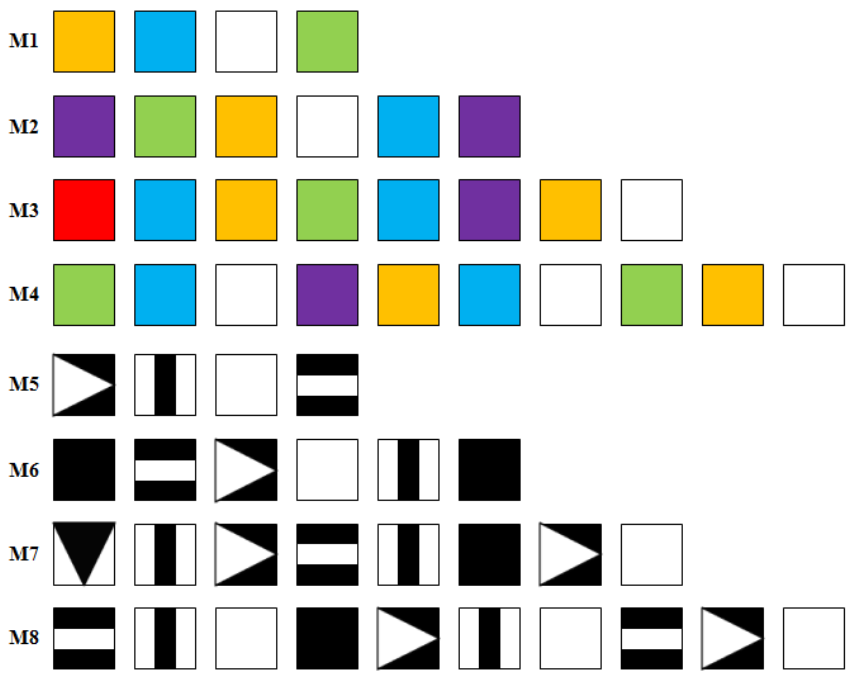

Figure 11: 8 TAG-Game ${ }^{\mathrm{M}}$ items.

\subsection{Computational Complexity Measures}

Difficulty is a relative attribute that can vary depending on a person's developmental status, age, and health conditions. For an assessment test to produce reliable and sensitive assays, it must be neither too difficult nor too easy. For TAG-Games to be potentially useful as a dynamic assessment tool, it was necessary that a computational measure of play complexity, $C$, be defined. This value, associated with each TAG-Game, allows the administrator to personalize each task to the test subject. Fig. 12 shows the complexity values calculated for the items in each TAG-Game.

\subsubsection{Complexity measure of TAG-Game ${ }^{A}$}

Based on an information-theoretic approach and adopting the concept of the parts entropy (Sanderson, 1984; Lee, Moses, \& Chirikjian, 2008), the play complexity associated with TAG-Game ${ }^{\mathrm{A}}$ is defined by the change in configurational entropy:

$$
C^{A}=H^{\text {initial }}-H^{\text {final }}
$$



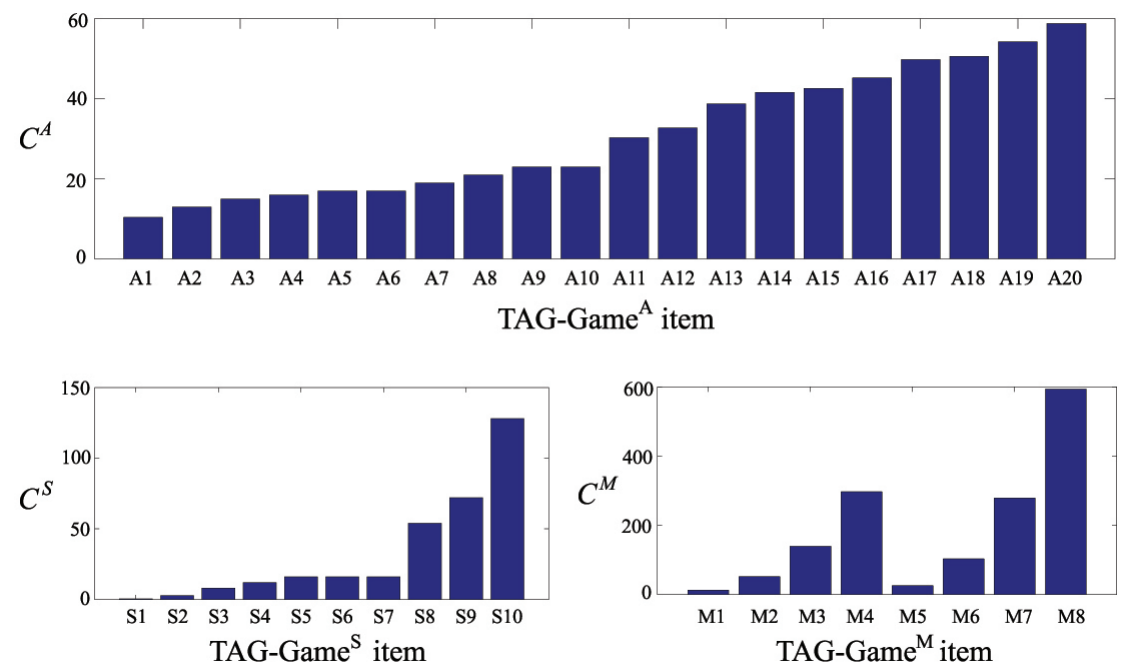

Figure 12: Calculated complexity values for all items in each TAG-Game, i.e., $C^{A}, C^{S}$, and $C^{M}$.

where $H^{\text {initial }}$ indicates the amount of randomness/uncertainty for the set of geometric blocks used in play and $H^{\text {final }}$ is the remaining randomness/uncertainly after successfully accomplishing a given task (e.g. assembling the blocks to form a specific assembly configuration), measured by the discrete entropy. Therefore, the difference between $H^{\text {initial }}$ and $H^{\text {final }}$ indicates the amount of uncertainty that is reduced by the person assembling the blocks.

\subsubsection{Complexity measure of TAG-Game ${ }^{S}$}

The play complexity of TAG-Game ${ }^{\mathrm{S}}$ takes into account the variety of pattern properties that increase or decrease the complexity of the item. The factors that increase the complexity are the total number of blocks, the number of unique blocks, and the length of the embedded pattern. The factors that decrease the complexity are the number of times the pattern repeats and the number of symmetry axes contained in the pattern. The play complexity of TAG-Game ${ }^{\mathrm{S}}$ is calculated as

$$
C^{S}=\frac{N \cdot N_{d} \cdot L}{R \cdot(S+1)}
$$

where $N$ is the total number of blocks, $N_{d}$ is the number of face images used in the pattern, $L$ is the pattern length, $R$ is the number of pattern repeats, and $S$ is the number of symmetry axes. 


\subsubsection{Complexity measure of TAG-Game ${ }^{M}$}

The difficulty in remembering a sequence of images depends on the complexity of the employed images, the number of images, and the number of elements repeated within the sequence. The complexity measure is defined by the configurational entropy of the entire sequence, computed by

$$
C^{M}=\log _{2} Q+\log _{2} L
$$

where $Q$ is the number of all possible arrangements for the given images and $\mathrm{L}$ is the sequence length. For example, if the sequence involves four colors, the number of their possible arrangements in four distinctive locations is $Q=4 !=24$ and the corresponding $C^{M}=\log _{2} 24+\log _{2} 4=6.585$. If the sequence item uses the same colored image four times, $Q=4 ! / 4 !=1$, such that $C^{M}=\log _{2} 1+\log _{2} 4=2$. If the geometric images used in TAGGame $^{\mathrm{A}}$ and TAG-Game ${ }^{\mathrm{S}}$ are used, the total number of possible arrangements is multiplied by the number of distinctive orientations for each image so that the complexity of the geometric pattern is taken into account.

\section{Evaluation Methods}

The human-subject study focused on evaluating the two hypotheses stated in Section 1. To do so, the study design was aimed at testing 1) preliminary reliability and consistency of TAG-Games data, 2) the computational complexity measures, and 3) preliminary validity of TAG-Games measures on measuring target cognitive skills. This study was reviewed and approved by Case Western Reserve University's Institutional Review Board (IRB).

\subsection{Participants}

Eighty-six students (42 males) aged 18 to 30 years $($ Mean $=21.6, \mathrm{SD}=$ 3.1) were recruited and participated in the study via convenience sampling, where the participants were selected because of their convenient accessibility and proximity to the researchers. 47 (31 males, 66\%) were from the Engineering School and 39 (11 males, 28\%) were from the School of Arts and Science. There was a significant relationship between gender and school where there were more males from the Engineering School $\left(\chi_{1}^{2}=12.16, p<0.01\right)$. The proportion of males and females in our sample from the School of Engineering match with the demographics of the school (68\% male population). The percentage of male participants from the School of Arts and Science (28\%) was 
lower than the demographics of the school (43\%) because several students participated in this study for course credit, and the majority of students enrolled in this course were female. Participants were recruited from the Case Western Reserve University campus, where emails and flyers were used to find volunteers. Participants were compensated with either a gift card or course credit.

All 86 participants were administered the three types of TAG-Games. 48 out of 86 took two subtests of the Wechsler Adult Intelligence Scale - 4th Edition (WAIS-IV) (i.e. Block Design (BD) and Matrix Reasoning (MR)) in addition to the three TAG-Games, and 25 out of 48 were administered the Digit Span (DS) subtest in addition to BD and MR of WAIS-IV and the three TAG-Games. Among 48 participants who took the three TAGGames and BD and MR subtests of the WAIS-IV, 9 (7 males) were from the Engineering School and 39 (11 males) were from the Arts and Science. Among 25 participants who were administered all six tests, 2 (1 male) were from the Engineering and 23 ( 7 males) were from the Arts and Science. The demographics of the study participants is summarized in Table 3.

\subsection{Protocol}

Three types of TAG-Games and three subtests of WAIS-IV (i.e., BD, MR, and DS) were administered in the order shown in Table 4. The three subtests of WAIS-IV were selected to assess cognitive skills similar to those tested by TAG-Games. Each participant was given a brief oral presentation at the beginning of each TAG-Game with a sample item. The three WAIS-IV subtests were administered using the standardized protocol (i.e. instruction, prompts, time limits, and discontinuation rules), outlined in the WAIS-IV manual. They were administered in the order $\mathrm{BD} \rightarrow \mathrm{MR} \rightarrow \mathrm{DS}$, immediately following the TAG-Games. BD involves 14 items requiring the participants to manipulate a given set of blocks to match the assembly configuration presented in a booklet. It discontinues with two consecutive scores of 0 . MD contains 26 items of colored matrix or visual patterns requiring the participants to guess and select the missing item in the pattern among 5 possible answers. The test discontinues after 3 consecutive scores of 0 . DS consists of three parts: Digit Span Forward (DSF), Digit Span Backward (DSB), and Digit Span Sequencing (DSS); they contain 48 items in total, with 16 items in each. The sets of digits were presented verbally to the participants and they recited them in forward, backward, and increasing orders. The participants were given two trials for each length of digit sequence, and the 
test was discontinued when both trials scored zeros. The entire assessment took approximately 60 minutes.

\subsection{Scoring Methods}

Scoring of TAG-Game ${ }^{\mathrm{A}}$ was similar to the method used in the WAIS-IV BD subtest, addressing accuracy, item complexity, and speed (i.e. time taken to complete the item). Since TAG-Games are not standardized tests, the participants' performance was analyzed to determine the score distribution in a fashion similar to that of WAIS-IV. For items 1-10, 50\% of the correct answers were made within 10 seconds, resulting in 3 points. The other $50 \%$ of the correct answers, which took more than 10 seconds, yielded 2 points. For items 11-20, one third of the correct answers were made within 20 seconds resulting in 7 points, another one third between 20 and 24 seconds resulting in 5 points, and the rest took more than 24 seconds, yielding 3 points. For every item, incorrect assembly resulted in zero points. The total available score for TAG-Game ${ }^{\mathrm{A}}$ is 100.

Scoring for TAG-Game ${ }^{\mathrm{S}}$ also takes into consideration both accuracy and speed. For items 1-5, 50\% of correct answers were recorded within 4 seconds, resulting in 3 points. Correct answers made after 4 seconds resulted in 2 points. For items $6-10$, one third of correct answers were made within 8 seconds, resulting in 7 points, another one third between 8 and 12 seconds resulting in 5 points, and the rest took more than 12 seconds yielding 3 points. For every item, incorrect shape matching resulted in zero points. The total available score for TAG-Game ${ }^{\mathrm{S}}$ is 50 .

Scoring for TAG-Game ${ }^{\mathrm{M}}$ was based solely on the correctness of memory; completion time was not factored into the score. The total possible score of 56 was derived from 2 sets of blocks of 4 possible lengths (i.e. 4, 6, 8, and 10). More specifically, the color and geometric pattern sequences yielded scores of 28 each, accounting for a total of 56 points.

\subsection{Data Analysis}

The data analysis strategy focused on establishing the preliminary psychometric integrity of the measure. To achieve this purpose, split-half and test-retest reliability were conducted using Spearman's correlations with $\alpha=$ 0.05. Once the reliability of the measure was established, the validity of the TAG-Games was examined by correlations with WAIS-IV. Finally, exploratory behavior analysis was carried out by estimating the number of 
rotational motions applied to the blocks and speed. The analysis was restricted to TAG-Game ${ }^{\mathrm{A}}$ data only, because TAG-Game ${ }^{\mathrm{S}}$ and TAG-Game ${ }^{\mathrm{M}}$ use only one SIG-Block and are therefore not ideal to observe behavioral differences. The algorithm for estimating the rotational motions was based on the threshold value and the local maxima and minima in the acceleration data. The accelerations caused by the user manipulation were different from those obtained from the impact of the block assembly.

\section{Evaluation Results}

\subsection{Split-Half and Test-Retest Reliability}

In an effort to provide preliminary data on the psychometric property of TAG-Game, split-half and test-retest reliability tests were conducted using Spearman correlations for each of the games. Since the items varied on $C$, it was necessary to equate the sets on this variable before conducting the split-half reliability. Our first split was based on an odd and even strategy; unfortunately the sets differed significantly in $C$ (i.e. 29.94 vs. 31.83 in TAG-Game $^{\mathrm{A}} ; 22.50$ vs. 42.54 in TAG-Game ${ }^{\mathrm{S}}$; and 114.14 vs. 261.74 in TAG-Game ${ }^{\mathrm{M}}$ ). Given the knowledge of the play complexity of each set, we then moved items strategically across the sets to equate them on complexity. As a result, the TAG-Game ${ }^{\mathrm{A}}$ items were divided into $\{1,3,5,8,9,12,14$, $16,17,19\}$ and $\{2,4,6,7,10,11,13,15,18,20\}$, the TAG-Game ${ }^{\mathrm{S}}$ items into $\{1,2,6,7,10\}$ and $\{3,4,5,8,9\}$, and the TAG-Game ${ }^{\mathrm{M}}$ items into $\{1,5,6,8\}$ and $\{2,3,4,7\}$. Using Spearman's correlation coefficient, the split-half reliability coefficients were found to be $r(10)=0.73(p=0.016)$ for TAG-Game ${ }^{\mathrm{A}}, r(5)=0.98(p=0.005)$ for TAG-Game ${ }^{\mathrm{S}}$, and $r(4)=1.0$ $(p<0.001)$ for TAG-Game ${ }^{\mathrm{M}}$. Of the 86 participants, 10 were administered TAG-Games at two different times, approximately two weeks apart. The testretest reliability was found to be $r(10)=0.72(p=0.19)$ for TAG-Game ${ }^{\mathrm{A}}$, $r(10)=0.62(p=0.05)$ for TAG-Game ${ }^{\mathrm{S}}$, and $r(10)=0.75(p=0.013)$ for TAG-Game $^{\mathrm{M}}$.

\subsection{Validity of Complexity Measures}

To evaluate the computational complexity measures, we investigated the correlation between the participants' performance and the complexity value of each item. For this, the mean time required for completing TAG-Game ${ }^{\mathrm{A}}$ was taken as the index of performance and used to examine the impact of $C^{A}$ on performance. For TAG-Game ${ }^{\mathrm{S}}$, incorrectness was computed by 
the normalized number of total incorrect answers for each item, and for TAG-Game ${ }^{\mathrm{M}}$ it was computed by the normalized number of total images that the player could not memorize, or could memorize only incorrectly, in each sequence item. Time for completion in TAG-Game ${ }^{\mathrm{A}}$ was highly correlated with $C^{A}$ where $r(20)=0.91(p<0.001)$, indicating that the more complex the assembly task, the longer the participants took to complete the design. Correctness on TAG-Game ${ }^{\mathrm{S}}$ was also highly correlated with $C^{S}$ where $r(10)=0.95(p<0.001)$. As regards TAG-Game ${ }^{\mathrm{M}}$, eight sequence items were examined and high correlation was found between the correctness and play complexity, $r(8)=0.83(p=0.01)$. For all the three TAG-Games, the selected performance measures fitted well to a linear function with the corresponding $R^{2}$ close to 1 .

\subsection{Relationship between TAG-Games and WAIS-IV Subtests}

Of the 86 participants who completed the three TAG-Games, 48 completed two subtests of WAIS-IV, BD and MR, and 25 also completed the DS subtest. The number of participants in the WAIS-IV subtests was smaller than the total number of participants, because the study was initially focused on evaluating the preliminary reliability of the TAG-Games and validity of complexity measures and only later became focused on the preliminary validity evaluation of target measures. We first began with two subtests, $\mathrm{BD}$ and $\mathrm{MR}$, and later added DS for correlational analysis. Correlations between the three TAG-Games and the three subtests of WAIS-IV are provided in Table 5. TAG-Game ${ }^{\mathrm{A}}$ and the BD subtest scores were correlated by $r(48)=0.48(p=0.001)$, and TAG-Game ${ }^{\mathrm{S}}$ and the MR subtest by $r(48)=0.45(p=0.001)$. Correlations were also found between TAG-Game ${ }^{\mathrm{S}}$ and $\mathrm{BD}(r(48)=0.39, p=0.006)$, TAG-Game ${ }^{\mathrm{M}}$ and $\mathrm{DS}(r(25)=0.33, .108)$, and TAG-Game ${ }^{\mathrm{M}}$ and BD $(r(48)=0.28, p=0.05)$. Weak correlations were found between TAG-Games ${ }^{\mathrm{A}}$ and TAG-Games ${ }^{\mathrm{S}}(r=0.25, p=0.023)$ and between TAG-Games ${ }^{\mathrm{A}}$ and TAG-Games ${ }^{\mathrm{M}}(r(86)=0.22, p=0.42)$. BD and MR were also correlated by $r(48)=0.38, p=0.007$.

\subsection{Exploratory Behavioral Analysis}

We performed additional analysis to explore the potential of the SIGBlocks system for behavioral assessment. The noisy acceleration data was filtered and processed into the discrete rotational motions applied to each block. For this exploratory analysis, TAG-Game ${ }^{\mathrm{A}}$ was selected because it involves multiple block manipulations while the other two games use only a 
single block, making them inappropriate for observing behavioral differences. The participants were divided into three groups based on their scores in TAGGame $^{\mathrm{A}}$ : the high-score, middle-score, and low-score groups. Five individuals from each of these three groups of TAG-Game ${ }^{\mathrm{A}}$ were randomly selected. For each, the speed of manipulative behavior and the total number of rotations applied to the blocks were automatically measured by an embedded algorithm. Fig. 13 shows an interesting finding. Although there were individual differences within the group, a positive correlation was found between the TAG-Game ${ }^{\mathrm{A}}$ score and the manipulation speed $(r(15)=0.81, p<0.001)$ (See Fig. 13a). On the other hand, as shown in Fig. 13b, no significant group difference was observed in the total number of rotations applied to the blocks during play $(r(15)=0.22, p=0.424)$.

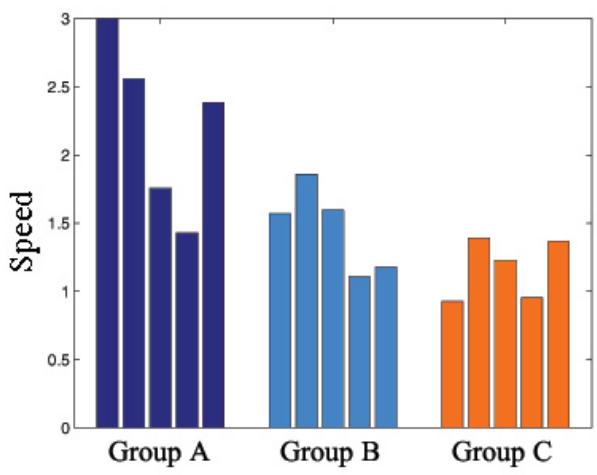

(a)

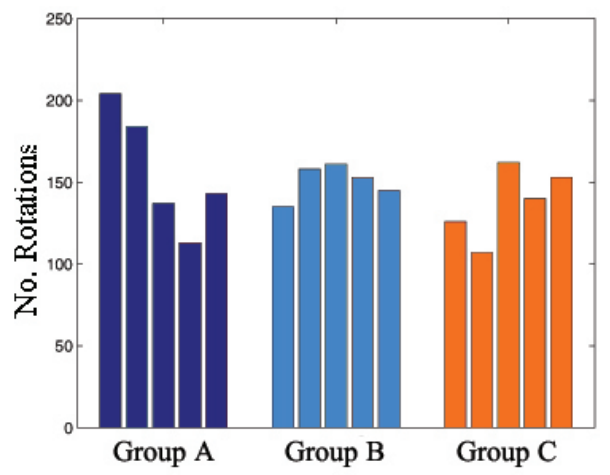

(b)

Figure 13: Speed of rotations for three groups (a) and total number of rotations (b). Fast decision and movement were recorded in the high-score group of TAG-Game ${ }^{\mathrm{A}}$.

\subsection{Discussion}

The present investigation suggests TAG-Games has the potential to be used as a cognitive assessment tool; however, the data here should be viewed with caution. The study was a first step towards establishing the reliability and validity of this measure. Small sample size, convenience sampling, and the use of the single administration order (i.e. three TAG-Games always followed by the WAIS-IV subtests) do not allow for generalization of the results and complete evaluation of this technology-based cognitive assessment method. Extended evaluation studies addressing these limitations 
must follow. In addition, while the presented technology aims to be a fully autonomous and remote assessment tool, the evaluation study involved an administrator to ensure proper use of the hardware, which is a lab-made prototype, and record sensor errors. The accuracy of the automated measurement of the time and accuracy at each manipulation step by the SIGBlocks was over 98\%. It was determined that the technical errors were mostly related to the contact failure in the optical sensors. Replacement of these connectors would significantly improve measurement accuracy.

With these caveats in mind, the SIG-Blocks technology is technically advanced and produces data which could not be captured in traditional humanadministered approaches. The blocks' unique communication strategies and real-time wireless assessment capabilities surpass the existing systems for the use in cognitive assessment. The SIG-Blocks system can also capture behavioral data in addition to the speed and accuracy for each test item, as described in Section 7.4. Further investigation will be required for using this technology for additional behavioral assessment. To assess behavior, objective and accurate measurement of target behavioral data is essential and the presented technology forms the technical groundwork by enabling such data collection in an objective and fully automated manner.

\section{Conclusion and Future Work}

A new tangible game interface technology, SIG-Blocks, and the computerized TAG-Games using the SIG-Blocks, TAG-Games, were developed and tested for their usefulness in cognitive assessment. The technology realizes wireless and real-time assessment of cognitive skills by employing a set of SIG-Blocks with embedded motion sensors. Three types of TAG-Games were developed for preliminary technical and human subject evaluations. Reliability of the TAG-Game items was assessed using split-half and test-retest reliability tests for evaluating internal consistency and stability over time. The results show high correlations in the evaluations by both of the tests. The defined measures of play complexity are also highly correlated with the participants' performance in each game, implying that the computational measures are potentially useful for generating dynamic test items meeting each player's performance and cognitive level. For preliminary assessment of the validity of TAG-Games for measuring target cognitive skills, we compared the TAG-Games data with widely accepted standardized measures (i.e. the BD, MR, and DS subtests of WAIS-IV). Moderate correlation was 
found between TAG-Game ${ }^{\mathrm{A}}$ and BD and TAG-Game ${ }^{\mathrm{S}}$. TAG-Game ${ }^{\mathrm{S}}$ and BD and TAG-Game ${ }^{\mathrm{M}}$ and DS were also correlated. Weak correlations were also found among the TAG-Games, such that $r=0.25$ between TAG-Games ${ }^{\mathrm{A}}$ and TAG-Games ${ }^{\mathrm{S}}$, and $r=0.22$ between TAG-Games ${ }^{\mathrm{A}}$ and TAG-Games ${ }^{\mathrm{M}}$. Among the WAIS-IV subtests, a correlation $(r=0.38)$ was found between $\mathrm{BD}$ and MR.

Building on the results presented in this paper, extended human subject studies will be performed for further validation of the TAG-Game measures. The future studies will consider (1) broadening of the ages of the participants, (2) randomizing participants if possible, and (3) randomizing the test order. There are also a few technical improvements to be made. Although the current GUI supports only 2-D assembly, modification to 3-D game expansion would broaden the scope of our research to cover various applications. In addition, the cover images on the blocks can be changed or the blocks may be equipped with liquid crystal display (LCD) or light emitting diode (LED) displays for generating diverse visual feedback. The three TAG-Games presented in this paper do not incorporate augmented visual graphics or animated features, which may be important for making these games more fun and engaging.

Once fully evaluated, the SIG-Blocks technology will have the potential to provide advanced instrumentation for various research, clinical, and educational applications. Such applications can be so diverse as to include clinical psychology, early-childhood education, and cognitive and motor skill assessments with automated, objective, cumulative and real-time data collection that enables remote and continuous assessments. Technology-based approaches, such as the presented work, can also address limitations in the traditional assessment methods by i) reducing cost by automating the process and thus avoiding clinician/administrator time, ii) improving the quantity and quality of the measurable data, iii) enabling objective assessment, iv) enabling wireless, remote administration for hard-to-reach areas, and v) providing a dynamic test, tailored for each individual or group to increase sensitivity in assays.

\section{References}

[1] Alers, S. H. M., \& Barakova, E. I. (2009). Multi-agent platform for development of educational games for children with autism. IEEE ICE CIG, 2009, 47-53. 
[2] Bernmark, E., \& Wiktorin, C. (2002). A triaxial accelerometer for measuring arm movements. Applied ergonomics, 33(6), 541-547.

[3] Bors, D. A.,\& Stokes, T. L. (1998). Raven's Advanced Progressive Matrices: Norms for first-year university students and the development of a short form. Educational and Psychological Measurement, 58(3), 382-398.

[4] Chen, H. C., \& Chang, L. Y. (2012). Design and implementation of a ZigBee-based wireless automatic meter reading system. PRZEGLAD ELEKTROTECHNICZNY (Electrical Review), 88(1b), 64-68.

[5] Christensen, H., Griffiths, K., MacKinnon, A., \& Jacomb, P. (1997). A quantitative review of cognitive deficits in depression and Alzheimertype dementia. Journal of the International Neuropsychological Society, 3(06), 631-651.

[6] Cubelets. Retrieved from http://www.modrobotics.com/.

[7] Fork, M., Bartels, C., Ebert, A. D., Grubich, C., Synowitz, H., \& Wallesch, C. W. (2005). Neuropsychological sequelae of diffuse traumatic brain injury. Brain injury, 19(2), 101-108.

[8] Jeong, D., Kerci, E., \& Lee, K. (2010, September). TaG-Games: tangible geometric games for assessing cognitive problem-solving skills and fine motor proficiency. In Multisensor Fusion and Integration for Intelligent Systems (MFI), 2010 IEEE Conference on (pp. 32-37). IEEE.

[9] Jeong, D., \& Lee, K. (2016). Distributed Communication and Localization Algorithms for Homogeneous Robotic Swarm. In Distributed Autonomous Robotic Systems (pp. 405-418). Springer Japan.

[10] Kulp, M. T. (1999). Relationship between visual motor integration skill and academic performance in kindergarten through third grade. Optometry $\&$ Vision Science, 76(3), 159-163.

[11] Lee, K., \& Jeong, D. (2014, October). Memorix: A tangible memory game using iSIG-Blocks. In Games Media Entertainment (GEM), 2014 IEEE (pp. 1-8). IEEE.

[12] Lee, K., Moses, M., \& Chirikjian, G. S. (2008). Robotic self-replication in structured environments: Physical demonstrations and complexity 
measures. The International Journal of Robotics Research, 27(3-4), 387401.

[13] Lezak, M. D. (2004). Neuropsychological assessment. Oxford University Press, USA.

[14] Lockman, J. J. (2000). A perceptionaction perspective on tool use development. Child development, 71(1), 137-144.

[15] Lund, H. H., \& Nielsen, C. B. (2011, November). Modularity for modulating exercises and levels-observations from cardiac, stroke, and COLD patients therapy. In Ubiquitous Robots and Ambient Intelligence (URAI), 2011 8th International Conference on (pp. 253-258). IEEE.

[16] McNerney, T. S. (1999). Tangible programming bricks: An approach to making programming accessible to everyone (Doctoral dissertation, Massachusetts Institute of Technology).

[17] Merrill, D., Sun, E., \& Kalanithi, J. (2012, May). Sifteo cubes. In CHI'12 Extended Abstracts on Human Factors in Computing Systems (pp. 10151018). ACM.

[18] Millis, S. R., Rosenthal, M., Novack, T. A., Sherer, M., Nick, T. G., Kreutzer, J. S., High, W. M., \& Ricker, J. H. (2001). Longterm neuropsychological outcome after traumatic brain injury. The Journal of head trauma rehabilitation, 16(4), 343-355.

[19] Pehoski, C., Henderson, A., \& Tickle-Degnen, L. (1997). In-hand manipulation in young children: translation movements. American Journal of Occupational Therapy, 51(9), 719-728.

[20] Petersen, R. C., Smith, G. E., Waring, S. C., Ivnik, R. J., Kokmen, E., \& Tangelos, E. G. (1997). Aging, memory, and mild cognitive impairment. International Psychogeriatrics, 9(S1), 65-69.

[21] Polatajko, H. J., \& Cantin, N. (2005, December). Developmental coordination disorder (dyspraxia): an overview of the state of the art. In Seminars in pediatric neurology (Vol. 12, No. 4, pp. 250-258). WB Saunders. 
[22] Raven, J. C., \& Court, J. H. (1998). Raven's progressive matrices and vocabulary scales. Oxford, UK: Oxford Psychologists Press.

[23] Sanderson, A. (1984, March). Parts entropy methods for robotic assembly system design. In Robotics and Automation. Proceedings. 1984 IEEE International Conference on (Vol. 1, pp. 600-608).

[24] Schoemaker, M. M., Niemeijer, A. S., Reynders, K., \& Smits-Engelsman, B. C. M. (2003). Effectiveness of neuromotor task training for children with developmental coordination disorder: a pilot study. Neural plasticity, 10(1-2), 155-163.

[25] Schweikardt, E., \& Gross, M. D. (2006, November). roBlocks: a robotic construction kit for mathematics and science education. In Proceedings of the 8th international conference on Multimodal interfaces (pp. 72-75). ACM.

[26] Sharlin, E., Itoh, Y., Watson, B., Kitamura, Y., Sutphen, S., \& Liu, L. (2002, April). Cognitive cubes: a tangible user interface for cognitive assessment. InProceedings of the SIGCHI conference on Human factors in computing systems (pp. 347-354). ACM.

[27] Short, E. J., \& Weissberg-Benchell, J. A. (1989). The triple alliance for learning: Cognition, metacognition, and motivation. In Cognitive strategy research (pp. 33-63). Springer New York.

[28] Silverman, W., Miezejeski, C., Ryan, R., Zigman, W., Krinsky-McHale, S., \& Urv, T. (2010). Stanford-Binet and WAIS IQ differences and their implications for adults with intellectual disability (aka mental retardation). Intelligence, 38(2), 242-248.

[29] Sparrow, S. S., \& Davis, S. M. (2000). Recent advances in the assessment of intelligence and cognition. Journal of Child Psychology and Psychiatry, 41(1), 117-131.

[30] Strauss, E., Sherman, E. M., \& Spreen, O. (2006). A compendium of neuropsychological tests: Administration, norms, and commentary. American Chemical Society.

[31] Suzuki, H., \& Kato, H. (1995, October). Interaction-level support for collaborative learning: AlgoBlockan open programming language. In 
The first international conference on Computer support for collaborative learning (pp. 349-355). L. Erlbaum Associates Inc.

[32] Tatemichi, T. K., Desmond, D. W., Stern, Y., Paik, M., Sano, M., \& Bagiella, E. (1994). Cognitive impairment after stroke: frequency, patterns, and relationship to functional abilities. Journal of Neurology, Neurosurgery \&3 Psychiatry, 57(2), 202-207.

[33] Terrenghi, L., Kranz, M., Holleis, P., \& Schmidt, A. (2006). A cube to learn: a tangible user interface for the design of a learning appliance. Personal and Ubiquitous Computing, 10(2-3), 153-158.

[34] Willingham, D. B., Koroshetz, W. J., \& Peterson, E. W. (1996). Motor skills have diverse neural bases: Spared and impaired skill acquisition in Huntington's disease. Neuropsychology, 10(3), 315.

[35] Wong, W. Y., \& Wong, M. S. (2008). Detecting spinal posture change in sitting positions with tri-axial accelerometers. Gait $\mathscr{E}^{\mathcal{S}}$ Posture, 27(1), 168-171.

[36] Wright, F., Rosenbaum, P. L., Goldsmith, C. H., Law, M., \& Fehlings, D. L. (2008). How do changes in body functions and structures, activity, and participation relate in children with cerebral palsy?. Developmental Medicine \& Child Neurology, 50(4), 283-289.

[37] Wyeth, P., \& Wyeth, G. F. (2001). Electronic blocks: Tangible programming elements for preschoolers. In IFIP TC. 13 International Conference on Human-Computer Interaction (Vol. 1, pp. 496-503). IOC Press. 


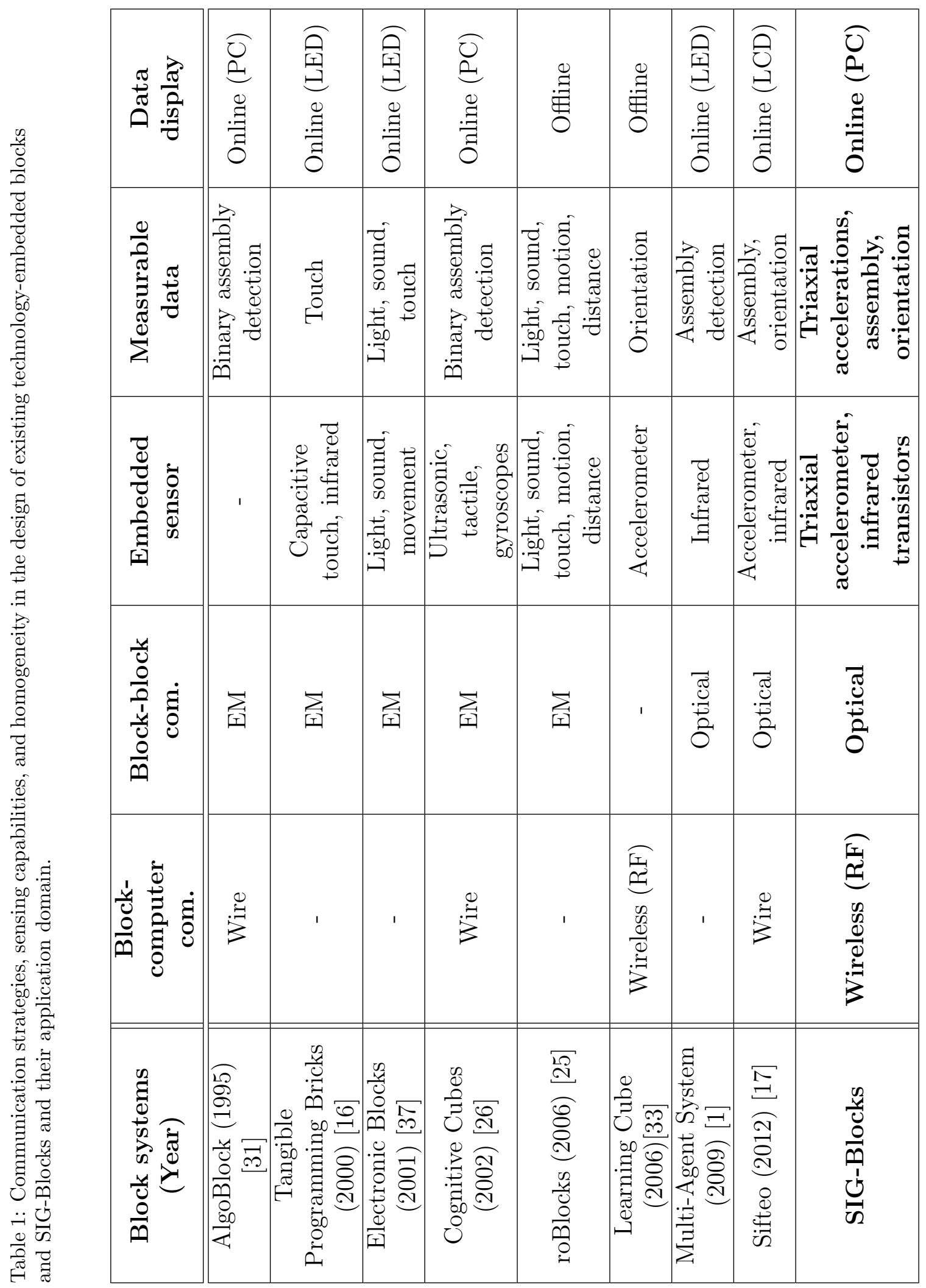


Table 2: Three types of games and the cognitive skills expected to be associated with each game.

\begin{tabular}{|c|l|}
\hline \multicolumn{1}{|c|}{ Type } & Associated cognitive skills \\
\hline TAG-Game $^{\mathrm{A}}$ & $\begin{array}{l}\text { Fine-motor proficiency, visual-motor integration, low-level } \\
\text { working memory, attention span }\end{array}$ \\
\hline TAG-Game $^{\mathrm{S}}$ & $\begin{array}{l}\text { Fine-motor proficiency, visual-motor integration, low-level } \\
\text { working memory, cognitive problem solving, attention span }\end{array}$ \\
\hline TAG-Game $^{\mathrm{M}}$ & $\begin{array}{l}\text { Fine-motor proficiency, visual-motor integration, high-level } \\
\text { working memory, attention span }\end{array}$ \\
\hline
\end{tabular}

Table 3: Demographics of the participants for the three TAG-Games (TAG-Game ${ }^{\mathrm{A}}$ (TA), TAG-Game $^{\mathrm{S}}$ (TS), and TAG-Game ${ }^{\mathrm{M}}(\mathrm{TM})$ ) and the three subtests of WAIS-IV. The table shows the number of participants, the number of female participants, and the average age with the standard deviation (SD).

\begin{tabular}{|cccccc|c|c|c|}
\hline TA & TS & TM & BD & MR & DS & No. Par. & No. Males & Ave. Age (SD) \\
\hline$\circ$ & $\circ$ & $\circ$ & $\times$ & $\times$ & $\times$ & 86 & 38 & $21.6(3.1)$ \\
\hline$\circ$ & $\circ$ & $\circ$ & $\circ$ & $\circ$ & $\times$ & 48 & 18 & $20.9(2.5)$ \\
\hline$\circ$ & $\circ$ & $\circ$ & $\circ$ & $\circ$ & $\circ$ & 25 & 8 & $20.4(1.1)$ \\
\hline
\end{tabular}

Table 4: The test involves six subtests $(60 \mathrm{~min})$, including three TAG-Games and three subtests of WAIS-IV.

\begin{tabular}{c|ccc}
\hline Order & Task & Time & No. Items \\
\hline 1 & TAG-Game $^{\mathrm{A}}(\mathrm{TA})$ & $15 \mathrm{~min}$ & 20 \\
2 & TAG-Game $^{\mathrm{S}}(\mathrm{TS})$ & $5 \mathrm{~min}$ & 10 \\
3 & TAG-Game $^{\mathrm{M}}(\mathrm{TM})$ & $15 \mathrm{~min}$ & 8 \\
4 & WAIS: BD & $5 \mathrm{~min}$ & 14 \\
5 & WAIS: MR & $10 \mathrm{~min}$ & 26 \\
6 & WAIS: DS & $10 \mathrm{~min}$ & 48 \\
\hline
\end{tabular}


Table 5: Correlations among the three TAG-Games and WAIS-IV subtests (BD, MR, and DS).

\begin{tabular}{|c|ccc|}
\hline & TA & TS & TM \\
\hline BD & $r(48)=\mathbf{0 . 4 8}$ & $r(48)=0.39$ & $r(48)=0.28$ \\
& $(p=0.001)$ & $(p=0.006)$ & $(p=0.05)$ \\
\hline MR & $r(48)=0.03$ & $r(48)=\mathbf{0 . 4 5}$ & $r(48)=0.09$ \\
& $(p=0.825)$ & $(p=0.001)$ & $(p=0.556)$ \\
\hline DS & $r(25)=-0.19$ & $r(25)=0.07$ & $r(25)=\mathbf{0 . 3 3}$ \\
& $(p=0.365)$ & $(p=0.74)$ & $(p=0.108)$ \\
\hline
\end{tabular}




\title{
SIG-Blocks: Tangible Game Technology for Automated Cognitive Assessment
}

\author{
Kiju Lee, Ph.D. \\ Corresponding Author \\ Assistant Professor in Mechanical and Aerospace Engineering \\ Case Western Reserve University \\ 10900 Euclid Avenue, Cleveland, OH 44106 \\ kiju.lee@case.edu \\ Tel: 216-368-2943 \\ Fax: 216-368-3007 \\ Donghwa Jeong, Ph.D. \\ Department of Mechanical and Aerospace Engineering \\ Case Western Reserve University \\ Donghwa.jeong@,case.edu \\ Rachael C. Schindler, Ph.D. Candidate \\ Department of Psychological Sciences \\ Case Western Reserve University \\ Rdc55@,case.edu \\ Elizabeth J. Short, Ph.D. \\ Professor in Psychological Sciences \\ Case Western Reserve University \\ Elizabeth.Short@case.edu
}

